\title{
OPERATORS QUASISIMILAR TO A NORMAL OPERATOR
}

\author{
CONSTANTIN APOSTOL ${ }^{1}$
}

ABSTRACT. A necessary and sufficient condition for an operator to be quasisimilar to a normal operator is given.

Let $X$ be a complex Banach space and let $T$ be a bounded linear operator acting in $X$. The aim of this note is to provide a necessary and sufficient condition for $T$ to be quasisimilar to a normal operator acting in a Hilbert space.

This problem was called to the attention of the author by R. G. Douglas.

Before stating our result, recall some basic definitions. Let $Y$ be a complex Banach space and let $A: Y \rightarrow X$ be a bounded linear operator. We shall call $A$ a quasiaffinity if it injective and has dense range. Let $S$ be a bounded linear operator acting in $Y$. We shall say that $T$ is quasisimilar with $S$ if there exist two quasiaffinities $A: Y \rightarrow X, B: X \rightarrow Y$, such that $T A=A S, S B=B T$. For more details see [1]. A finite or countable system $\left\{X_{n}\right\}_{1 \leq n<m}$ of subspaces on $X$ will be called basic if, for any $n$, the subspaces $X_{n^{*}} \operatorname{cl} m\left\{X_{k}\right\}_{k \neq n}$ are complementary and $\bigcap_{1 \leq n<m}\left(\operatorname{cl} m\left\{X_{k}\right\}_{k \geq n}\right)=\{0\}$ if $m=\infty$.

We shall prove the following

Theorem. The operator $T \in \mathfrak{L}(X)$ is quasisimilar to a normal operator if and only if there exists a basic system $\left\{X_{n}\right\}_{1 \leq n<m}$ of invariant subspaces of $T$ such that $T \mid X_{n}$ is similar to some normal operator.

Proof. Sufficiency。 Let $\left\{X_{n}\right\}_{1 \leq n<m}$ be a basic system of invariant subspaces of $T$ such that $T \mid X_{n}$ is similar to some normal operator $S_{n}$ acting in the Hilbert space $H_{n}$. Since we have

$$
\left\|S_{n}\right\|=\left|S_{n}\right|_{s p}=\left|\left(T \mid X_{n}\right)\right|_{s p} \leq\left\|\left(T \mid X_{n}\right)\right\| \leq\|T\|,
$$

we may define the normal operator $S=\bigoplus_{1 \leq n<m} S_{n}$ acting in $H=\bigoplus_{1 \leq n<m} H_{n^{\circ}}$. Let $C_{n}: H_{n} \rightarrow X_{n}$ be an invertible operator such that $T \mid X_{n}=C_{n} S_{n} C_{n}^{-1}$, and let $P_{n}$ be the canonical projection of $X$ onto $X_{n}$ determined by the decomposition $X=X_{n}+\operatorname{cl} m\left\{X_{k}\right\}_{k \neq n}$. Choosing positive numbers $\alpha_{n}, \beta_{n} \neq 0$,

Received by the editors September 3, 1974. $47 \mathrm{~B} 40$.

AMS (MOS) subject classifications (1970). Primary 47B99; Secondary 47A65,

Key words and phrases. Quasimilarity, normal operator.

1 This research was done while the author was a Visiting Scholar at State University of New York at Stony Brook, by an agreement between the Romanian Academy of Sciences and the National Academy of Sciences. 
such that

$$
\sum_{1 \leq n<\mathrm{m}} \alpha_{n}\left\|C_{n}\right\|<\infty, \quad \sum_{1 \leq n<m} \beta_{n}\left\|C_{n}^{-1}\right\|\left\|P_{n}\right\|<\infty
$$

we may define the quasiaffinities $A: H \rightarrow X, B: X \rightarrow H$, by the equation

$$
\begin{aligned}
A\left(\bigoplus_{1 \leq n<m}^{\bigoplus} h_{n}\right) & =\sum_{1 \leq n<m} a_{n} C_{n} h_{n}, \quad h_{n} \in H_{n}, \\
B x & =\bigoplus_{1 \leq n<m} \beta_{n} C_{n}^{-1} P_{n} x, \quad x \in X .
\end{aligned}
$$

But it is easy to see that we have $T A=A S, S B=B T$; thus $T$ is quasisimilar with $T$.

Necessity. Suppose that $T$ is quasisimilar to a normal operator $S$ acting in a Hilbert space $H$. Let $A: H \rightarrow X, B: X \rightarrow H$ be two quasiaffinities such that $T A=A S, S B=B T$. Since obviously $S$ commutes with $B A$, applying Fuglede's theorem we infer that $S$ commutes with the spectral measure $E$, of the modulus of $B \Lambda$. We consider the two possible cases:

(i) $B . A$ is not invertible,

(ii) $B A$ is invertible.

(i) If $B A$ is not invertible, then 0 is an accumulation point in the spectrum of the modulus of $B A$; thus we can find a strictly decreasing sequence $\left\{a_{n}\right\}_{n=1}^{\infty}$, such that $a_{1}=\|B A\|, \lim _{n \rightarrow \infty} a_{n}=0, E\left(\left[a_{n}, a_{n+1}\right)\right) \neq 0$. Let us put

$$
H_{n}=E\left(\left[a_{n}, a_{n+1}\right)\right) H, \quad S_{n}=S \mid H_{n} .
$$

Since the restriction of $B A$ to $H_{n}$ is bounded from below by $a_{n}$, the restriction of $A$ to $H_{n}$ will be bounded from below; thus $X_{n}=A H_{n}$ is a closed subspace in $X$. The relations

$$
T X_{n}=T A H_{n}=A S H_{n} \subset A H_{n}=X_{n}
$$

show that $X_{n}$ is an invariant subspace of $T$. If we define $C_{n}: H_{n} \rightarrow X_{n}$ to be the restriction of $A$ to $H_{n}$, then it is easy to see that we have $T \mid X_{n}=$ $C_{n} S_{n} C_{n}^{-1}$, thus $T \mid X_{n}$ is similar to a normal operator. Let us put $\sigma_{n}=$ $\left[0, a_{1}\right) \backslash\left[a_{n}, a_{n+1}\right)$. Since obviously we have $\operatorname{cl} m\left\{X_{k}\right\}_{k \neq n}=\operatorname{cl} m\left\{A E\left(\sigma_{n}\right) H\right\}$, using the relations

$$
\begin{array}{r}
\left\|A\left(h_{n}+h_{n}^{\prime}\right)\right\|^{2} \geq\|B\|^{-2}\left\|B A\left(h_{n}+h_{n}^{\prime}\right)\right\|^{2}=\|B\|^{-2}\left(\left\|B A h_{n}\right\|^{2}+\left\|B A h_{n}^{\prime}\right\|^{2}\right) \\
\geq\|B\|^{-2} a_{n}^{2}\left\|h_{n}\right\|^{2} \geq\|A\|^{-2}\|B\|^{-2} a_{n}^{2}\left\|A h_{n}\right\| \\
(\forall) h_{n} \in H_{n}, h_{n}^{\prime} \in E\left(\sigma_{n}\right) H,
\end{array}
$$


we obtain that the angle between $X_{n}$ and $\operatorname{cl} m\left\{X_{k}\right\}_{k \neq n}$ is positive and we have

$$
X_{n}+\operatorname{clm}\left\{X_{k}\right\}_{k \neq n}=\operatorname{cl} m\{A H\}=X, \bigcap_{n=1}^{\infty}\left(\operatorname{cl} m\left\{X_{k}\right\}_{k \geq n}\right)=\{0\} .
$$

This shows that the system $\left\{X_{n}\right\}_{n=1}^{\infty}$ is basic.

(ii) If $B A$ is invertible, $A$ results to be invertible; thus $T$ is similar with $S$ and we can take the basic system $\left\{X_{k}\right\}_{1 \leq n<m}$, with $m=2, X_{1}=X$.

Remark. An examination of the proof of our Theorem shows that we can replace the word "normal" in the statement by either "selfadjoint" or "unitary".

\section{REFERENCES}

1. C. Foiaş, C. Pearcy and D. Voiculescu, The staircase repres entation of biquasitriangular operators, Michigan Math. J. (to appear).

2. T. B. Hoover, Quasi-similarity of operators, Illinois J. Math. 16 (1972), 678686. MR $47 \# 866$.

3. B. Sz.-Nagy and C. Foiaş, Analyse harmonique des opérateurs de l'espace de Hilbert, Masson, Paris; Akad. Kiadó, Budapest, 1967; English reve transl., NorthHolland, Amsterdam; American Elsevier, New York; Akad. Kiadó, Budapest, 1970. MR $37 \# 778 ; 43 \# 947$.

DEPARTMENT OF MATHEMATICS, STATE UNIVERSITY OF NEW YORK AT STONY BROOK, STONY BROOK, NEW YORK 11794

Current address: Institute of Mathematics, Romanian Academy of Sciences, Bucharest, Romania 